\title{
A Rainfall-Based Model for Predicting the Regional Incidence of Wheat Seed Infection by Stagonospora nodorum in New York
}

\author{
Denis A. Shah and Gary C. Bergstrom
}

Department of Plant Pathology, Cornell University, Ithaca, NY 14853.

Current address of D. A. Shah: 5179 Tuscarora Rd., Niagara Falls, NY 14304.

Accepted for publication 22 January 2002.

\begin{abstract}
Shah, D. A., and Bergstrom, G. C. 2002. A rainfall-based model for predicting the regional incidence of wheat seed infection by Stagonospora nodorum in New York. Phytopathology 92:511-518.

Our goal was to develop a simple model for predicting the incidence of wheat seed infection by Stagonospora nodorum across western and central New York in any given year. The distribution of the incidence of seed infection by $S$. nodorum across the region was well described by the beta-binomial probability distribution (parameters $p$ and $\theta$ ). Mean
\end{abstract}

ABSTRACT monthly rainfalls in May and in June across western and central New York were used to predict $p$. The binary power law was used to predict $\theta$. The model was validated with independent data collected from New York. The predicted distribution of seed infection incidence was not statistically different from the actual distribution of the incidence of seed infection.

Additional keywords: gamma distribution, generalized bootstrap, multiple regression.
Much careful mathematical attention has been paid to describing plant disease epidemics at the field scale, whereas the description of epidemics at the regional scale has remained mostly qualitative or summarized as surveys. Unique combinations of inoculum availability, host susceptibility, and microscale environmental conditions lead to variability in epidemics among fields. However, there is an added component of variability in epidemics because of factors (mainly environmental) occurring at the mesoscale. Mesoscale factors may account for much of the variability in epidemics occurring across a region, so that 1 year is not described as an epidemic year, whereas the following year may see more severe epidemics as a whole. Quantifying the variability in epidemics at the mesoscale level is a necessary step toward predicting the risk of severe epidemics across a region.

Stagonospora nodorum blotch (SNB) is a common disease of wheat (Triticum aestivum L.) caused by the fungus Stagonospora nodorum (Berk.) Castellani \& E. G. Germano (syn. Septoria nodorum (Berk.) Berk. in Berk. \& Broome; teleomorph Phaeosphaeria nodorum (E. Müller) Hedjaroude, syn. Leptosphaeria nodorum E. Müller). Winter wheat in New York is grown mainly in the western and central counties. Agronomic practices in New York preclude infected wheat debris as an important source of Stagonospora nodorum inoculum, because the crop is grown in a 2 year or greater rotation with corn, alfalfa, oat, soybean, and vegetable crops (all nonhosts to $S$. nodorum) on relatively small, spatially discontinuous fields. The teleomorph has never been positively identified in New York. Seed infection by $S$. nodorum is frequent in New York wheat seeds (21) and exceeds $50 \%$ in some seed lots. Seed transmission occurs at high frequency (70\% or greater) to the coleoptiles (22), which may be partially exposed above the soil line. Transmission also occurs to the first leaves at frequencies of 2 to $37 \%$ (22), and possibly occurs to other leaves as well. Therefore, infected seeds can be a source of primary inoculum for SNB in New York.

Corresponding author: G. C. Bergstrom; E-mail address: gcb3@cornell.edu

Publication no. P-2002-0314-01R

(c) 2002 The American Phytopathological Society
Rainfall is crucial to SNB development because pycnidiospores of $S$. nodorum are dispersed in splash droplets. For there to be any chance of seed infection, pycnidiospores must be splashed upwards from infected foliage to the wheat spikes, which are held above the canopy by the peduncle. Because vertical splashing occurs over a few centimeters (20), the pathogen must be sporulating at a height in the canopy that would enable spore bearing splash droplets to reach the spikes. Thus, rainfall during stem elongation (so that SNB may progress vertically in the developing canopy) as well as rainfall during the seed developmental stages (for splashing onto the spikes) may both be important factors affecting the incidence of wheat seed infection by $S$. nodorum.

Certified wheat seed lots in New York are harvested in July and are sown by grain producers as soon as 6 weeks later, allowing little time for the completion of seed assays for the incidence of $S$. nodorum. Our goal was to develop a simple model that could be used to predict the regional distribution in the incidence of wheat seed infection by $S$. nodorum shortly after harvest, or even before, without having to wait on the results of seed assays. The objective of this research was to model the relationship between the incidence of wheat seed infection by $S$. nodorum and rainfall across western and central New York.

\section{MATERIALS AND METHODS}

The incidence of seedborne $S$. nodorum in New York winter wheat has been surveyed since 1990. Samples of seeds from lots produced in New York and submitted for certification testing were obtained from the New York State Seed Testing Laboratory (New York Agricultural Experiment Station, Geneva). Testing of wheat seed lots for infection by $S$. nodorum is not part of the certification process. Therefore, samples submitted to the seed testing laboratory may be regarded as random with respect to $S$. nodorum infection; moreover, lots with high levels of seed infection by $S$. nodorum are often healthy otherwise (24). The number of lots assayed varied from year to year (Table 1), depending on how many samples were received from the testing laboratory. Samples were stored at $5^{\circ} \mathrm{C}$ until they could be assayed for seed infection (usually within 1 to 4 months). The incidence of seed infection by 
$S$. nodorum does not decrease during this length of time at low temperatures (4). The incidence of seed infection by $S$. nodorum was determined by assaying a minimum of 100 arbitrarily selected seeds per lot on a selective medium (19).

Probability distribution for seed infection incidence. When several seed lots from a region are tested, the mean seed infection incidence across all lots is usually reported and sometimes the range (which may be fairly wide or narrow) is also reported. However, there is the chance that some incidences will not be represented by the lots sampled from the region. Also, the mean and range do not indicate the likelihood of sampling a lot with a specified seed infection incidence. Probability distributions describing the incidence of infection not only convey the same information as summary statistics, such as the mean, but also offer the advantage of allowing one to estimate the chance of a given seed infection incidence for a particular seed lot.

Seed infection status is a binary variable (either an individual seed is infected by $S$. nodorum or it is not). Therefore, the binomial distribution is a reasonable starting point in fitting a discrete probability distribution to the incidence of wheat seed infection by $S$. nodorum. This probability distribution assumes that the mean incidence of infection is constant for all lots. However, differences in local environment together with differential cultivar susceptibility to seed infection by $S$. nodorum (23) could result in a nonconstant mean incidence of seed infection across lots. Therefore, the variation in seed infection incidence among seed lots (e.g., across western and central New York) would be greater than the expected variation assuming a binomial distribution fit the incidence of seed infection. This extra variation is referred to as overdispersion (2). A first step is to test whether data sets show overdispersion in the incidence of seed infection. Data sets were tested for overdispersion by calculating the binary form of the index of dispersion $(D)(15)$ for the number of seed positive for $S$. nodorum per lot. If $D>1$, then seed infection incidence may be overdispersed (contains variation in excess of that expected with the binomial distribution). The statistic $(N-1) \cdot D$ has a $\chi^{2}$ distribution (with $N-1 \mathrm{df}$, where $N$ is the number of seed lots in the sample) under the null hypothesis that incidence is distributed binomially, which allows a formal test for overdispersion (15).

The beta-binomial distribution is suitable for modeling overdispersed disease incidence data $(17,26,27,31)$. The distribution may be expressed in several formats (15), with one form being

$P(X=x)=\left(\begin{array}{c}n \\ x\end{array}\right) \operatorname{Beta}\{x+p / \theta, n-x+[(1-p) / \theta]\} / \operatorname{Beta}[p / \theta,(1-p) / \theta]$

where, in the present context, $P(X=x)$ is the probability that the random variable $X$ has the value $x, x$ is the number of seeds out of $n$ infected by $S$. nodorum, and $\operatorname{Beta}(a, b)$ is the beta function with parameters $a$ and $b$. The parameter $p$ is the expected value of the probability of a seed being infected, and $\theta$ is an index that increases with the amount of overdispersion. Whereas mean incidence of infection is assumed to be a constant for all lots with the binomial distribution, the beta-binomial allows for a mean incidence that varies from lot to lot.

The beta-binomial distribution was fitted to the incidence of seed infection by $S$. nodorum in each year. The $\chi^{2}$ goodness-of-fit (gof) test was used to assess the fit of the distribution to the observed data. For $\chi^{2}$ gof tests, seed infection incidence levels were pooled into bins (e.g., 0 to $5 \%$ ) so that expected frequencies were greater than five. The $\chi^{2}$ gof test was not possible for the 1999 data because there were insufficient degrees of freedom after pooling (9). Evaluating gof with the 1995 data set was problematic because different bin sizes led to different interpretations of gof with the $\chi^{2}$ test, illustrating an inherent weakness of the test. Therefore, for the 1995 and 1999 data sets, a dithered KolmogorovSmirnov (K-S) gof test was used (29). This test is based on the traditional K-S gof test for continuous distributions, but the test statistic is modified so that the tables for continuous distributions may be used for discrete distributions as well. A disadvantage of the K-S test is that software is not available for obtaining exact $P$ values as with the $\chi^{2}$ gof test; goodness-of-fit is assessed by comparing the test statistic to critical levels that are generally tabulated for only a few significance values. Tests for overdispersion and $\chi^{2}$ gof tests were done with the BBD software program (14). The dithered K-S gof test was done in Microsoft Excel 98 (Microsoft Corp., Redmond, WA).

Relating mean seed infection to rainfall. Wheat seeds are susceptible to infection by $S$. nodorum from flowering until the end of the dough period (5). Inoculum availability and temperature are unlikely limiting factors for seed infection; SNB is a polycyclic disease, seed infection occurs toward the end of the epidemic, and temperatures during seed development are favorable to the pathogen's growth (6).

We started with the premise that rainfall during stem elongation, and then rainfall from flowering to the end of seed maturity, were the two most important variables governing the incidence of wheat seed infection by $S$. nodorum. Data were not available on the locations of seed production fields or the dates of the beginning of stem elongation and flowering. However, in New York, the beginning of stem elongation generally coincides with the first week of May, and flowering starts about the first week of June. Seed development and maturation continues until the end of June. Seed infection should be a function of vertical splash droplet dispersal during rain. Yet commonly available meteorological measure-

TABLE 1. Incidence of seed infection by Stagonospora nodorum in winter wheat seed lots produced in western and central New York, 1990 to 1999 , and statistics for assessing the fit of the beta-binomial distribution to the data ${ }^{\mathrm{a}}$

\begin{tabular}{|c|c|c|c|c|c|c|c|c|}
\hline \multirow[b]{2}{*}{ Year $^{\mathrm{b}}$} & \multirow[b]{2}{*}{ No. of seed lots } & \multicolumn{2}{|c|}{ Seed infection $(\%)^{\mathrm{c}}$} & \multirow[b]{2}{*}{$D^{\mathrm{d}}$} & \multicolumn{2}{|c|}{ Goodness-of-fit test } & \multicolumn{2}{|c|}{ Beta-binomial parameters } \\
\hline & & Mean & Range & & $P_{\text {gof }}{ }^{\mathrm{e}}$ & $\mathrm{df}^{\mathrm{f}}$ & $p$ & $\theta$ \\
\hline 1990 & 50 & 22.5 & $1-71$ & 38.1 & 0.36 & 3 & 0.23 & 0.19 \\
\hline 1991 & 50 & 2.4 & $0-19$ & 11.0 & 0.05 & 1 & 0.02 & 0.05 \\
\hline 1995 & 70 & 4.5 & $0-42$ & 22.0 & $<0.01^{\mathrm{g}}$ & NA & 0.05 & 0.12 \\
\hline 1996 & 94 & 25.2 & $2-77$ & 18.6 & 0.05 & 4 & 0.26 & 0.16 \\
\hline 1998 & 26 & 10.4 & $0-40$ & 12.0 & 0.12 & 1 & 0.10 & 0.10 \\
\hline
\end{tabular}

a No data on seed infection were available for the years 1992, 1993, and 1997.

${ }^{\mathrm{b}}$ Data from 1990 and 1991 were published previously (21).

${ }^{c}$ Percentage of seeds infected was determined by assaying samples of at least 100 seeds per lot on a selective medium (19).

${ }^{\mathrm{d}}$ Index of dispersion. $P<0.0001$ in all years, meaning that the incidence of seed infection by $S$. nodorum across the western and central New York region was overdispersed.

${ }^{\text {e }} P$ value associated with the $\chi^{2}$ goodness-of-fit (gof) test for fit of the beta-binomial distribution to the data.

${ }^{\mathrm{f}}$ Degrees of freedom associated with the $\chi^{2}$ goodness-of-fit test for fit of the beta-binomial distribution to the data. Degrees of freedom are not applicable (NA) to the dithered Kolmogorov-Smirnov goodness-of-fit test, which is a nonparametric test.

${ }^{g} P$ value is for the dithered Kolmogorov-Smirnov goodness-of-fit test (29). 
ments such as rainfall amount, duration, or rate are not accurate predictors of splash height distribution, and rainfalls with similar characteristics may differ considerably in the splashing they cause (25). Thus, estimating the daily progression of SNB vertically in the canopy using daily rainfall measurements is a difficult task. In light of these limitations, and in keeping with our goal of developing a simple, practical model, we interpreted monthly rainfall as a summation of all possible dispersal events in that time period, regardless of their magnitude. Therefore, as a very general simplification, we used total rainfall during May as an estimate of the amount of rain on the crop during stem elongation, and total rainfall during June as an estimate of the amount of rain during seed development. We did not know the actual amount of rain received in May and June by the fields from which the seed lots were harvested, because we did not know the locations of those fields. However, these amounts can be estimated if we know the variability in the amount of rain received in May and June across the western and central New York region.

We obtained the rainfall data recorded at weather stations across the 16 western and central New York counties, which together account for over $90 \%$ of winter wheat acreage. The number of stations with available data varied between 41 and 54. The rainfalls recorded at these stations were regarded as a sample of all rainfall events that occurred throughout the region. We assumed that the samples were representative of the total distribution in rainfall across the region, and that probability models could describe this distribution. The gamma distribution is a probability model often used to describe rainfall (30), and was fitted to rainfall totals in May and June. The distribution appears in slightly different forms depending on the text consulted. We fitted gamma $(\phi, \omega)$, where the probability density function is defined as

$$
f(x)=\frac{w^{-\phi} x^{\phi-1} e^{-x / w}}{\Gamma(\phi)} \quad \text { if } x>0, \text { and } f(x)=0 \text { otherwise }
$$

where $x$ is monthly rainfall (millimeters), $\phi$ is the shape parameter $(\phi>0)$, and $\omega$ is the scale parameter $(\omega>0)$. The VTFIT software program (R. Cooke, University of Illinois, Urbana) was used to fit the distributions. The K-S gof test was used to determine the adequacy of the fit (3).

Preliminary plots were made of the distributions in the incidence of seed infection overlayed with the distributions in May and June rainfall. Visual inspection of the plots indicated that mean seed infection incidence $(n p)$ might be related to mean rainfall in May and mean rainfall in June. Multiple regression equations were used to relate $n p$ to mean rainfall in both May and June. Different equations with May and June rainfall as the predictor variables were tried. The form of the equation that predicted $n p$ best was

$$
n p_{i j}=\lambda_{0}+\lambda_{1} \operatorname{May}_{i}+\lambda_{2} \ln \left(\text { June }_{j}\right)+\varepsilon_{i j}
$$

where $\mathrm{May}_{i}$ is the mean rainfall (millimeters) in May, June $\mathrm{j}_{j}$ is the mean rainfall in June (millimeters), $\lambda_{0}, \lambda_{1}$, and $\lambda_{2}$ are regression parameters to be estimated, and $\varepsilon_{i j}$ is the associated error term. Since we were interested in modeling percent seed infection, $n$ was set at 100 . Mean monthly rainfall was estimated by the sample mean, since it is equivalent to the moment estimate of the mean of the gamma distribution (13).

Data on the incidence of wheat seed infection by $S$. nodorum in New York were available for 7 years only (Table 1). Because least square parameter estimates have a bias inversely proportional to sample size, there is reason for concern with such a small data set. As a solution, we generated data sets for the regression analysis by a method known as the generalized bootstrap (10). It differs from the familiar bootstrap (7) in that samples are generated from probability distributions fitted to the original data rather than sampling from the observed data itself. The bootstrap is not capable of generating samples containing observations that may be rare because it is based on the actual observed data, but this drawback was overcome by the generalized bootstrap, which is based on the probability distribution assumed to describe the population from which the actual sampled data were obtained. For the multiple regression, we generated 1,000 generalized bootstrap samples for each year (7,000 total samples) from the probability distributions fitted to seed infection incidence and rainfall. Mathematica (version 3.0, Wolfram Research, Inc., Champaign, IL) notebooks were written to generate the generalized bootstrap samples. Regression modeling was done in SAS (version 6.12, SAS Institute Inc., Cary, NC).

Binary power law. Once the parameter $p$ of the beta-binomial distribution was predicted, the next step was to obtain a prediction of $\theta$. In phytopathosystems examined to date, the binary power law (8) has been a good descriptor of the relationship between the observed variance in disease incidence and the expected variance, assuming incidence is described by the binomial distribution $(15,18,28)$. The binary power law and the beta-binomial distribution are linked, which allows the parameter $\theta$ of the beta-binomial to be estimated from the binary power law relationship (16).

We first determined whether the binary power law held for the incidence of seed infection by $S$. nodorum. Again, the generalized bootstrap was used to generate 1,000 samples from each of the 7 years, using the fitted beta-binomial distributions to incidence. The binary power law equation relating the variance in the observed number of infected seeds per sample to the variance expected with the binomial distribution is as follows:

$$
v_{\mathrm{obs}}=A_{x} v_{\mathrm{bin}}^{b}
$$

where $v_{\mathrm{obs}}$ is the observed variance in the number of seeds infected by $S$. nodorum per lot, and $v_{\text {bin }}$ is the variance assuming the number of infected seeds per lot is distributed binomially. $A_{x}$ and $b$ are parameters that can be estimated by linear regression after linearizing equation 4 by taking logarithms. If $b=1$ and $A_{x}>1$ (or $\left.\ln \left(A_{x}\right)>0\right)$, then $\theta$ does not vary with $p$. If both $A_{x}$ and $b$ are greater than 1 , then $\theta$ varies with $p$, which means that the degree of overdispersion among seed lots changes with the mean incidence of $S$. nodorum (16).

If the binary power law relationship holds, then the beta-binomial parameter $\theta$ can be estimated from

$$
\theta=\{a-[f(p) / n]\} /[f(p)-a]
$$

where $f(p)=[p(1-p)]^{1-b}$ and $a=A_{x} n^{b-2}$ (28).

Model verification and validation. The most definitive test of validity is to establish that the model's output is close to the output expected from the real system (13). The model was first checked to make sure it was giving an output (predicted $p$ and $\theta$ of the beta-binomial) that was realistic. Plots of the actual distribution of seed infection incidence were compared with the predicted distributions for the 7 years in Table 1. As a further check, the model was run with rainfall input from 1980 to 1988, 1992, 1993, and 1997. Seed infection data were not available for those years, but we wanted to make sure that the model's output probability distributions for seed infection incidence were within the limits of what would have been expected given the distribution of rainfall across western and central New York in May and June.

Validation is as much an art as it is a science (11). No standard theory on validation exists, but there is a set of general guidelines (1). There is strong interest in statistical validation techniques because they are reproducible and objective (12). In reality, the amount of data available for any modeling project varies greatly, and the availability of data on the real system determines which type of statistical technique can be used for validation (12). 

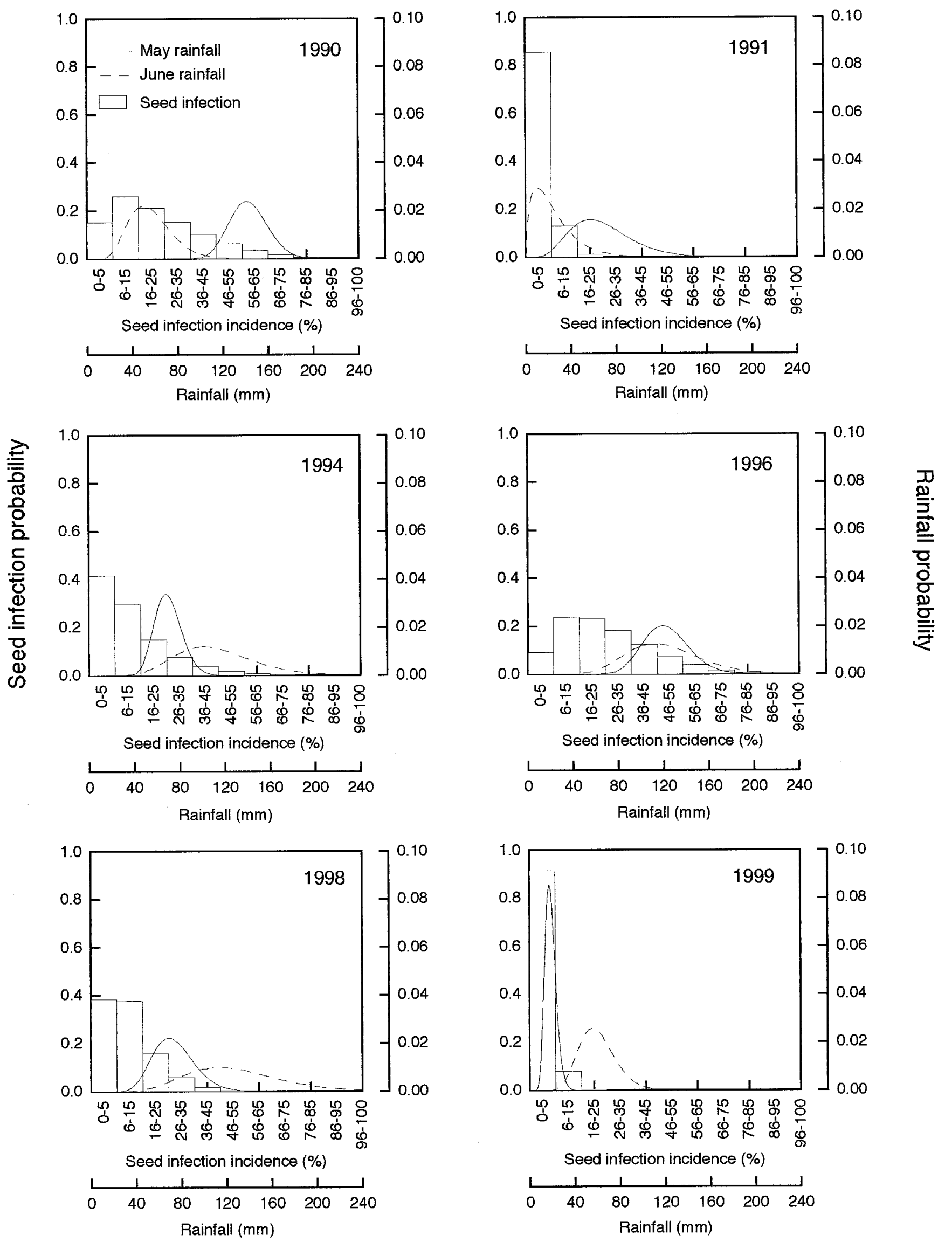

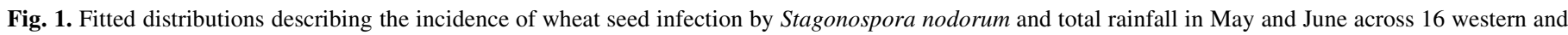

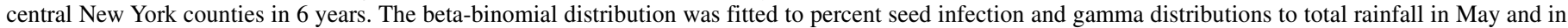
June. The $y$ axes represent probabilities of a given seed infection incidence range ( $x$ axis) or total monthly rainfall (offset $x$ axis). 
Statistical validation. One independent data set, consisting of the incidence of seed infection by $S$. nodorum determined for 279 lots produced in New York in 2000, was used for validation purposes. Mean monthly rainfall in May and June 2000, determined from the records at 38 weather stations across western and central New York, were used to predict the beta-binomial parameters $p$ and $\theta$. The predicted distribution of seed infection incidence was compared with the actual data by the $\chi^{2}$ gof test.

However, rainfall recorded at the 38 stations across western and central New York in 2000 is only one (assumed representative) sample of the distribution of rainfall in May and June. If rainfall had been recorded at different locations and used as model input, would the predicted parameters of the beta-binomial distribution still describe the actual data? To answer this question, we used a confidence interval approach. First, the beta-binomial distribution was fitted to the year 2000 data. The fit of the distribution was assessed by the $\chi^{2}$ gof test. The gamma distribution was then fitted to rainfall recorded in May and June 2000 at the 38 stations across central and western New York. The $\chi^{2}$ gof test was used to assess the adequacy of the fit of the gamma distribution. We did not know the actual rainfall during stem elongation and during seed development at the fields represented by the 279 sampled lots. We, however, assumed that the rainfalls at those fields and at the weather stations were realizations of the same population described by gamma distributions. One thousand generalized bootstrap samples were generated, each of size 38, using the fitted gamma distributions to May and June rainfall totals in 2000. Mean monthly rainfalls were calculated from each of the 1,000 samples, and served as the model's input rainfall variables. The predicted parameters $\left(\left[p_{i}, \theta_{i}\right], i=1, \ldots, 1,000\right)$ of the beta-binomial distribution describing seed infection incidence were used to calculate 95\% confidence intervals for $\left(p_{i}-p_{a}\right)$ and $\left(\theta_{i}-\theta_{a}\right)$, where $p_{a}$ and $\theta_{a}$ are the parameters of the beta-binomial distribution fitted to the actual seed infection incidence data in 2000. If zero is included in the confidence interval, then we conclude that the difference between the observed and predicted parameters is not significantly different at the $95 \%$ level. If zero is not included in the confidence interval, this does not necessarily invalidate the model as a practical representation of the system; it depends on the magnitude of the confidence interval (13).

\section{RESULTS}

Probability distribution for seed infection incidence. There was significant overdispersion in all data sets tested $(P<0.0001)$. The beta-binomial distribution was a reasonable fit to the observed data, except in 1995 (Table 1). The lack of fit in 1995 was mainly due to overprediction by the beta-binomial at zero incidence of infection.

Relating mean seed infection to rainfall. The gamma distribution fit monthly rainfall across western and central New York in May and in June $(P>0.05)$. Plots of the beta-binomial distributions fitted to seed infection incidence were overlayed with the fitted gamma distributions describing rainfall in May and in June (Fig. 1). Although the distributions of seed infection incidence were similar in some years, the underlying rainfall distributions could be quite different (Fig. 1).

The patterns revealed by the plots led to the hypothesis that the mean incidence of seed infection ( $n p)$ may be related to the combined effects of mean rainfall in May and June. Equation 3 fit the generalized bootstrap samples very well $\left(R^{2}=0.91\right)$ and was therefore considered suitable for representing the relationship between mean seed infection incidence and rainfall. Estimates for $\lambda_{0}, \lambda_{1}$, and $\lambda_{2}$ were $-26.10,0.19$, and 5.34, respectively. All three parameters were significantly different from zero $(P<0.0001)$.

Binary power law. Once the beta-binomial parameter $p$ could be specified from rainfall, the next step was to estimate the parameter $\theta$. Equation 4 was fitted by least squares regression to generalized bootstrap samples of seed lots. The regression was significant $\left(R^{2}=0.95\right)$. Estimates of $\ln \left(A_{x}\right)$ and $b$ were 1.28 and 1.52 , respectively. The $t$ tests indicated that $\ln \left(A_{x}\right)$ and $b$ were significantly greater than 0 and 1 , respectively $(P<0.0001)$, indi-
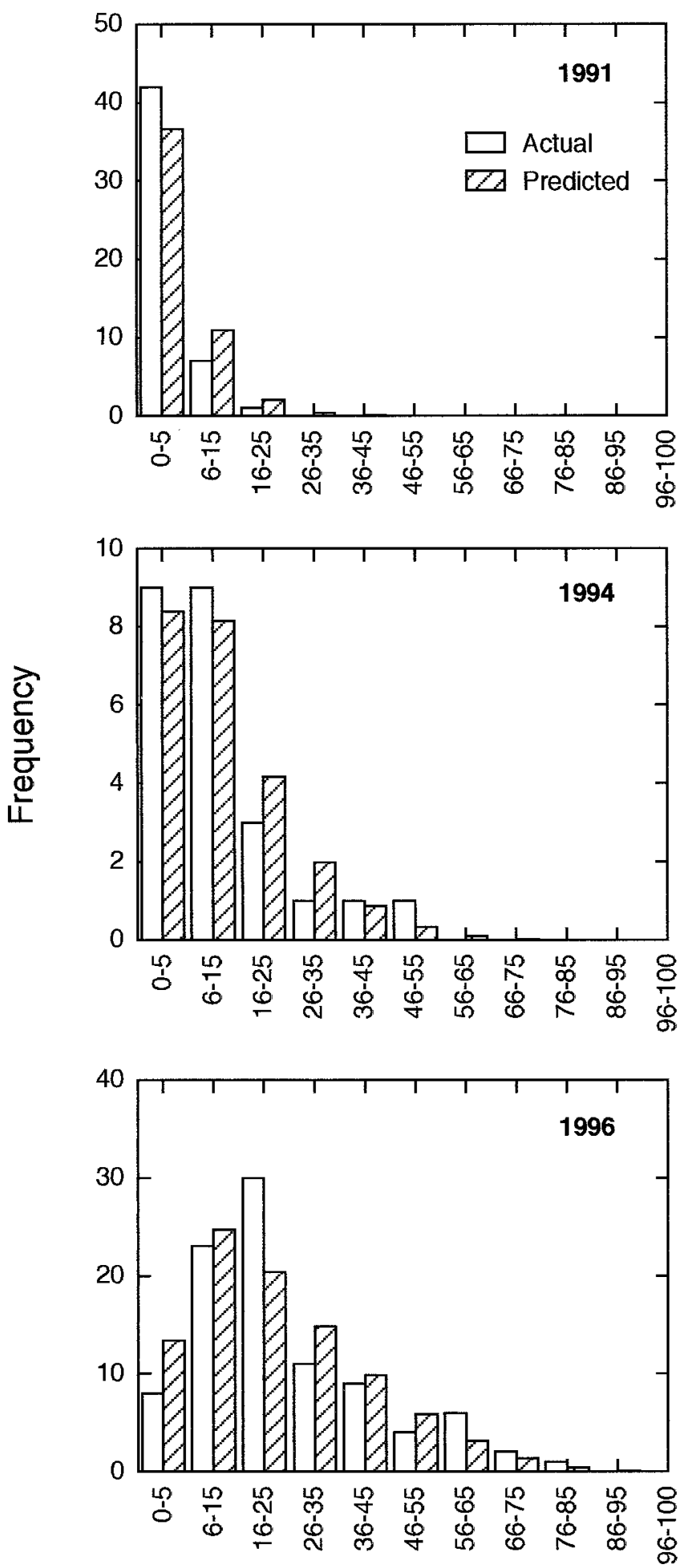

\section{Seed infection incidence (\%)}

Fig. 2. Actual and model predicted frequencies of the incidence of seed infection by Stagonospora nodorum in winter wheat seed lots across western and central New York in 1991, 1994, and 1996. Predicted frequencies were obtained from model derived parameters $(p, \theta)$ of the beta-binomial distribution. Predicted parameters were $(0.041,0.069),(0.125,0.133)$, and $(0.230,0.181)$ for 1991,1994 , and 1996 , respectively. 


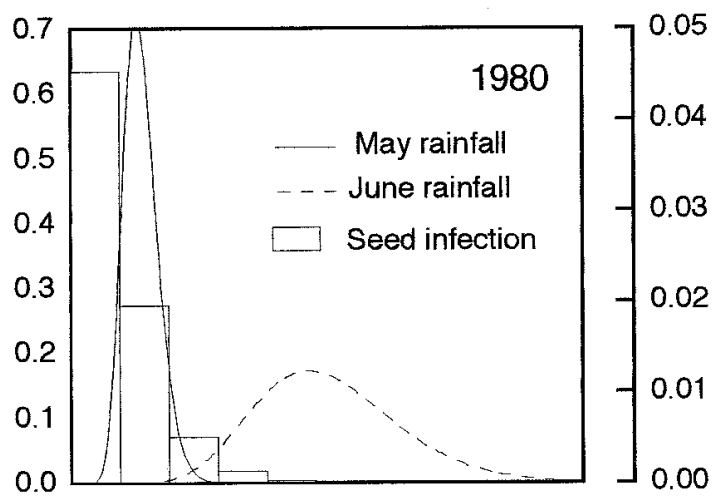

눙 눈

ó Seed infection incidence (\%)
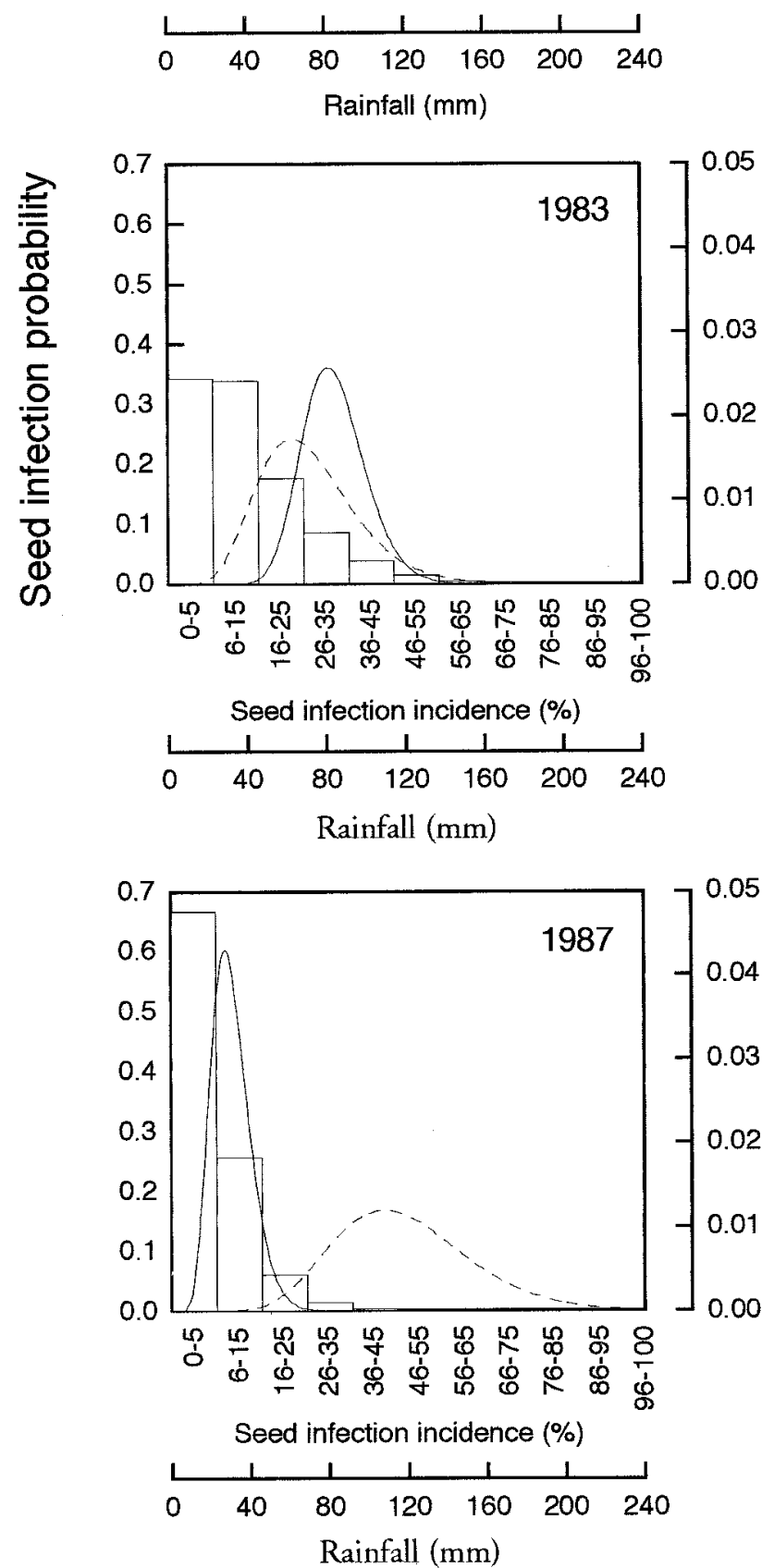

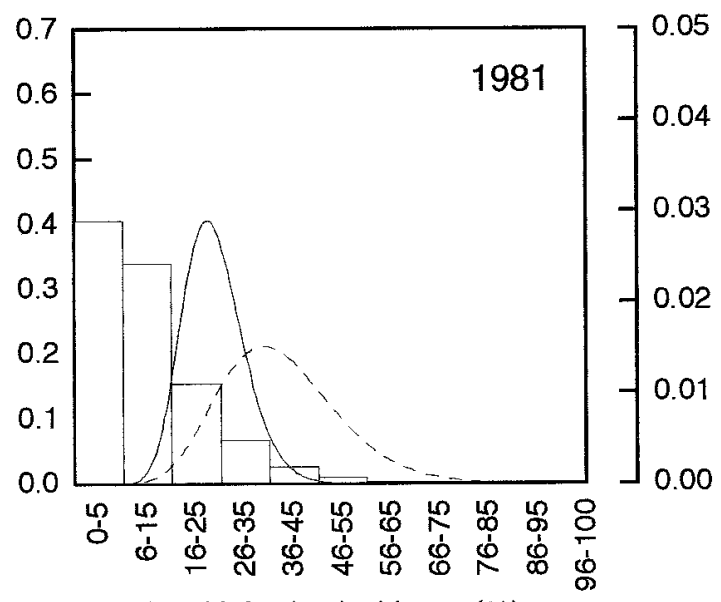

Seed infection incidence (\%)
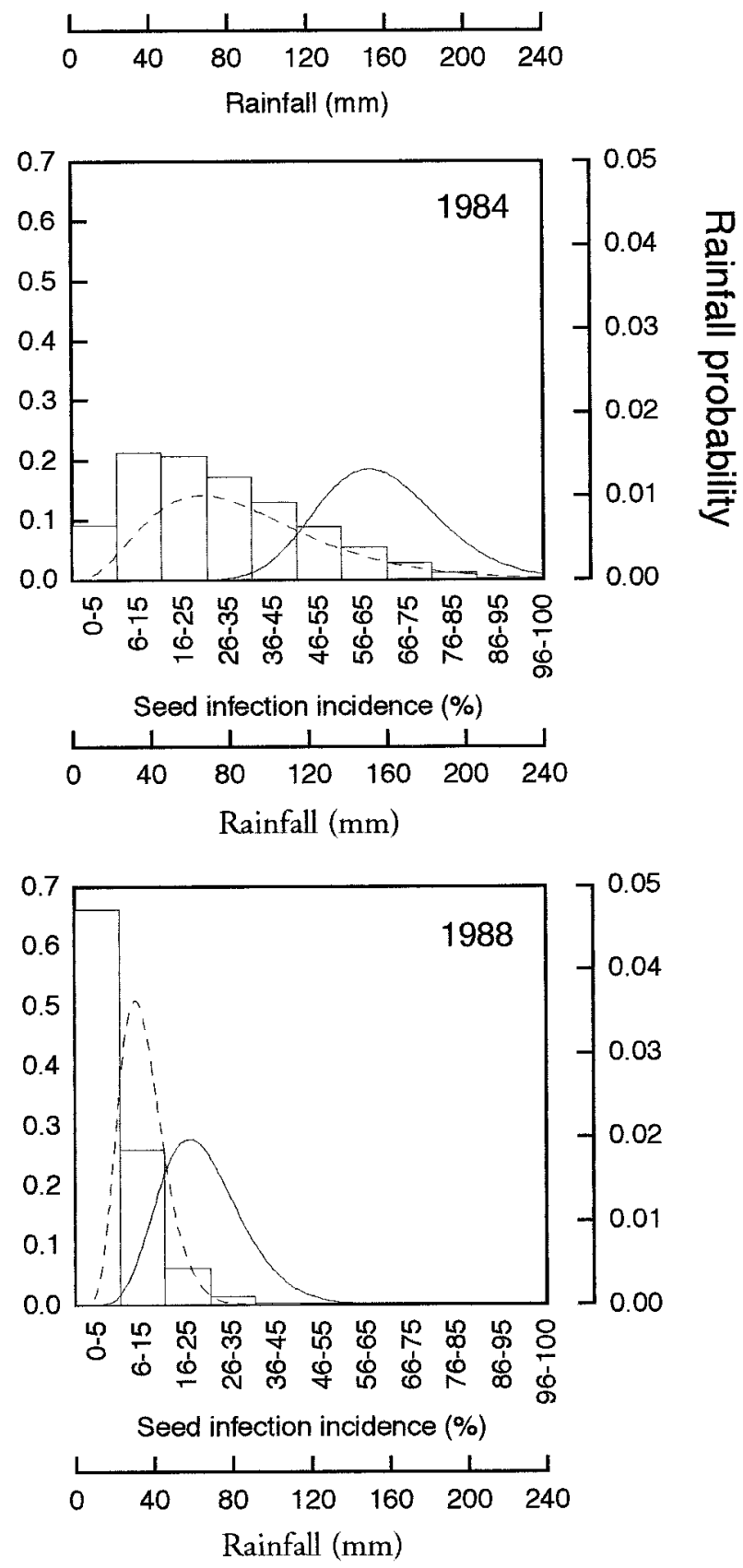

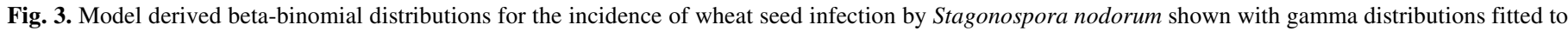
monthly total rainfall in May and in June across 16 western and central New York counties, 1980 to 1988. 
cating that the parameter $\theta$ varied with $p$. The parameter $\theta$ could now be predicted from equation 5 .

Model verification and validation. The actual and predicted distributions of seed infection incidence for the 7 years in Table 1 were quite close (Fig. 2), except for 1995. The model did give a negative value in 1999 for $p(=-0.0066)$. The model's output probability distributions for 1980 to $1988,1992,1993$, and 1997 appeared reasonable and corresponded to what would be expected given the rainfall patterns in those years (Fig. 3).

Statistical validation. Model-predicted beta-binomial parameters $(p, \theta)$, based on mean rainfall in May and June across western and central New York in 2000 , were $(0.233,0.182)$. The distribution of seed infection incidence based on the model-predicted parameters was not significantly different from the actual distribution in seed infection incidence $\left(P=0.50, \chi^{2}\right.$ gof test, $\left.23 \mathrm{df}\right)$. Actual and model-predicted frequencies for seed infection incidence for the 279 sampled lots are shown in Figure 4.

The beta-binomial distribution described actual seed infection incidence in $2000(P=0.57)$. The parameters $p_{a}$ and $\theta_{a}$ were estimated at 0.245 and 0.127 , respectively. The gamma distribution fit monthly rainfall in May $(P=0.58,6 \mathrm{df})$ and June $(P=0.45,7 \mathrm{df})$ in 2000 . Fitted parameters $(\phi, \omega)$ were $(38.34,3.18)$ for total rainfall in May and $(35.32,3.72)$ for total rainfall in June.

The $95 \%$ confidence interval for $\left(p_{i}-p_{a}\right)$ was $(-0.025,0.001)$, and was $(0.050,0.060)$ for $\left(\theta_{i}-\theta_{a}\right)$. Thus, model-derived predictions of $p$ are close to, and include, the actual value, because $0 \in$ $\left(p_{i}-p_{a}\right)$. Model predictions for $\theta$ are, however, positively biased. Nevertheless, the bias is fairly small and its range is very narrow.

\section{DISCUSSION}

Our objective was to develop a simple model for predicting the distribution in the incidence of wheat seed infection by $S$. nodorum across western and central New York from rainfall. To do this, we first needed a probability distribution that adequately described seed infection levels across the region. The beta-binomial distribution was a suitable probability distribution for this purpose. The beta-binomial distribution is described by two parameters $(p, \theta)$. Mean rainfalls in May and in June across western and central New York were used to predict $p$. Next, the binary power law was used to predict $\theta$. The predicted distribution of seed infection incidence in 2000 was not statistically different from the actual distribution of the incidence of seed infection that year.

It was possible to explain the distributions in the incidence of seed infection by the combined effects of rainfall during May and June (which were assumed to correspond to rainfall during stem elongation and then after flowering). For example, the distributions in seed infection incidence in 1991 and 1999 were quite similar. In 1991, it would appear that there was enough rain in May for the upward movement of SNB in the canopy, but very low rainfall in June would not have allowed for much splashing to occur from the canopy to the spikes during seed development. The situation appeared to have been different in 1999. In this year, there was very little rain in May, and so SNB would not have had many chances for progressing vertically in the canopy. Therefore, although rainfall in June was similar to other years in which there was high seed infection (e.g., 1990), the lack of vertical disease progression during stem elongation would have meant that the spikes were mainly beyond the reach of splash dispersal of $S$. nodorum pycnidiospores.

The incidence of seed infection by $S$. nodorum in 1 year was not related to the incidence in the previous year. For example, 1999 was a year with very low seed infection levels, but 2000 was a year in which seed infection incidences were relatively high, again supporting our assumption of rainfall as a major determinant of seed infection by $S$. nodorum in New York. Assuming that seedborne inoculum is the primary source for epidemics of SNB in New York and given that SNB is polycyclic, it follows that very low levels of initial inoculum (i.e., seed infection) can start potentially serious epidemics. Attempting to control SNB epidemics initiated from seedborne inoculum by treating seeds with fungicides against $S$. nodorum may be futile, not because of fungicidal inefficiency but because a few escapes may be sufficient to start the epidemic. Counterintuitively, the only time a seed treatment may actually reduce foliar epidemics substantially would be in years in which the incidence of seed infection in the sown seed was already very low, as in 1999. Under such circumstances, seed treatment against $S$. nodorum may come closest to true eradication of seedborne inoculum. This hypothesis requires testing.

We now have a way of estimating the probability of a given seed infection incidence level for a particular seed lot produced in western and central New York in any year. Furthermore, the longterm probabilities associated with any seed infection incidence can now be determined by driving the model with historic weather data. Results presented in this paper indicated that in most years the majority of lots contained less than $10 \%$ of seeds infected by $S$. nodorum. Also, a probability distribution describing the incidence of seed infection by $S$. nodorum is important in modeling seed to seedling transmission in the epidemiology of SNB.

One drawback of the model was that it predicted a negative value for mean probability of seed infection in 1999. Yet, seed infection incidences in that year were among the lowest we had observed since beginning routine seed assays. Viewed another way, the model was correct in predicting that seed infection incidences would have been very low in 1999. As more data on seed infection levels and rainfall are gathered, they should be incorporated into the model to allow more accurate estimation of regression and binary power law parameters. Other predictors of mean probability of seed infection, such as the number of days with rain during stem elongation and flowering, could be investigated. The model's structure should make future modifications a fairly easy task.

Ultimately, one may be interested in site-specific predictions of the incidence of seed infection. Site-specific predictions of seed infection would require estimates of the dates of key phenological stages as well as data on rain intensity and duration in individual seed production fields. A geographical information system may be of value in implementing such a model once the parameters have been established.

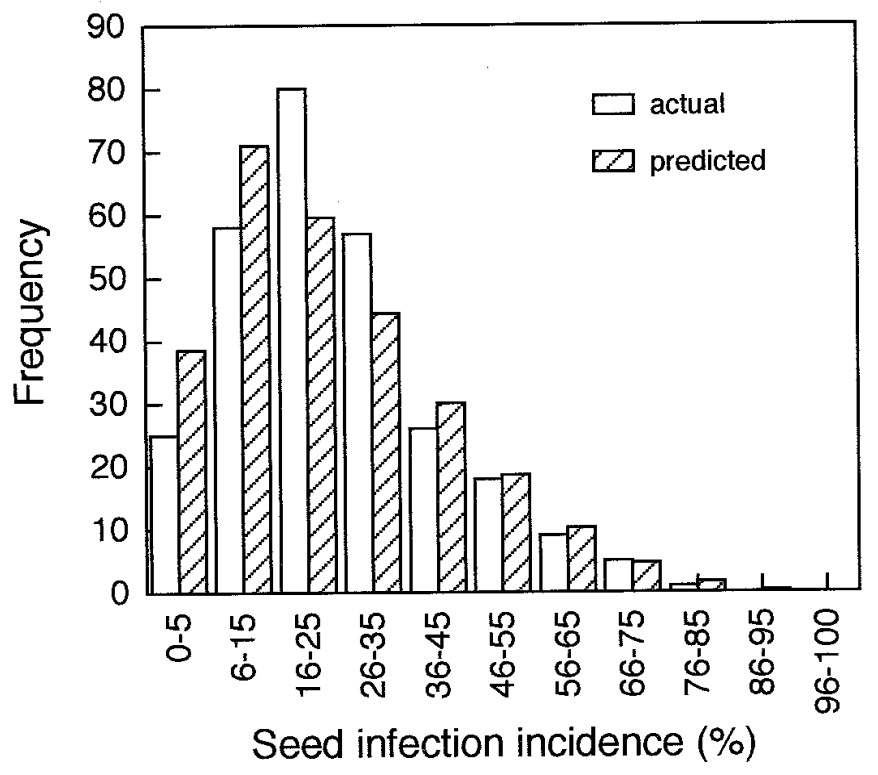

Fig. 4. Actual and model-predicted frequencies of the incidence of wheat seed infection by Stagonospora nodorum for 279 seed lots produced across western and central New York in 2000. Model-derived beta-binomial parameters $(p, \theta)$ were $(0.233,0.182)$. 


\section{ACKNOWLEDGMENTS}

This research was supported in part by Cornell University Hatch Project NYC153472 and by a graduate research assistantship to D. A. Shah from the Department of Plant Pathology, Cornell University.

\section{LITERATURE CITED}

1. Balci, O. 1997. Principles of simulation model validation, verification, and testing. Trans. Soc. Comput. Simul. 14:3-12.

2. Collett, D. 1991. Modelling Binary Data. Chapman \& Hall, London.

3. Conover, W. J. 1980. Practical Nonparametric Statistics. 2nd ed. John Wiley \& Sons, New York.

4. Cunfer, B. M. 1981. Survival of Septoria nodorum in wheat seed. Trans. Br. Mycol. Soc. 77:161-164.

5. Cunfer, B. M., and Johnson, J. W. 1981. Relationship of glume blotch symptoms on wheat heads to seed infection by Septoria nodorum. Trans. Br. Mycol. Soc. 76:205-211.

6. Djurle, A., and Yuen, J. E. 1991. A simulation model for Septoria nodorum in winter wheat. Agric. Syst. 37:193-218.

7. Efron, B., and Tibshirani, R. J. 1993. An Introduction to the Bootstrap. Chapman \& Hall, New York.

8. Hughes, G., and Madden, L. V. 1992. Aggregation and incidence of disease. Plant Pathol. 41:657-660.

9. Hughes, G., and Madden, L. V. 1993. Using the beta-binomial distribution to describe aggregated patterns of disease incidence. Phytopathology 83:759-763.

10. Karian, Z. A., and Dudewicz, E. J. 2000. Fitting Statistical Distributions: The Generalized Lambda Distribution and Generalized Bootstrap Methods. CRC Press, Boca Raton, FL.

11. Kleijnen, J. P. C. 1995. Case study: Statistical validation of simulation models. Eur. J. Oper. Res. 87:21-34

12. Kleijnen, J. P. C. 1995 . Verification and validation of simulation models. Eur. J. Oper. Res. 82:145-162.

13. Law, A. M., and Kelton, W. D. 2000. Simulation Modeling and Analysis. 3rd ed. McGraw-Hill Publishing, Boston.

14. Madden, L. V., and Hughes, G. 1994. BBD—Computer software for fitting the beta-binomial distribution to disease incidence data. Plant Dis. 78:536-540.

15. Madden, L. V., and Hughes, G. 1995. Plant disease incidence: Distributions, heterogeneity, and temporal analysis. Annu. Rev. Phytopathol. 33:529-564.
16. Madden, L. V., and Hughes, G. 1999. Sampling for plant disease incidence. Phytopathology 89:1088-1103.

17. Madden, L. V., Hughes, G., and Ellis, M. A. 1995. Spatial heterogeneity of the incidence of grape downy mildew. Phytopathology 85:269-275.

18. Madden, L. V., Nault, L. R., Murral, D. J., and Apelt, M. R. 1995. Spatial pattern analysis of the incidence of aster yellows disease in lettuce. Res. Popul. Ecol. 37:279-289.

19. Manandhar, J. B., and Cunfer, B. M. 1991. An improved selective medium for the assay of Septoria nodorum from wheat seed. Phytopathology 81:771-773.

20. Pietravalle, S., van den Bosch, F., Welham, S. J., Parker, S. R., and Lovell, D. J. 2001. Modelling of rain splash trajectories and prediction of rain splash height. Agric. For. Meteorol. 109:171-185.

21. Shah, D., and Bergstrom, G. C. 1993. Assessment of seedborne Stagonospora nodorum in New York soft white winter wheat. Plant Dis. 77:468471.

22. Shah, D. A., and Bergstrom, G. C. 2000. Temperature dependent seed transmission of Stagonospora nodorum in wheat. Eur. J. Plant Pathol. 106:837-842.

23. Shah, D. A., Bergstrom, G. C., and Sorrells, M. E. 2000. Differential seed infection of wheat cultivars by Stagonospora nodorum. Plant Dis. 84:749-752.

24. Shah, D. A., Bergstrom, G. C., and Sorrells, M. E. Relationship between wheat seed infection by Stagonospora nodorum and seed weight. Seed Sci. Technol. In Press.

25. Shaw, M. W. 1991. Variation in the height to which tracer is moved by splash during natural summer rain in the UK. Agric. For. Meteorol. 55:1-14.

26. Smith, M., Page, W., Holt, J., and Kyetere, D. 2000. Spatial dynamics of maize streak virus disease epidemic development in maize fields. Int. J. Pest Manag. 46:55-66.

27. Turechek, W. W., and Madden, L. V. 1999. Spatial pattern analysis of strawberry leaf blight in perennial production systems. Phytopathology $89: 421-433$

28. Turechek, W. W., and Madden, L. V. 1999. Spatial pattern analysis and sequential sampling for the incidence of leaf spot on strawberry in Ohio. Plant Dis. 83:992-1000.

29. Washburn, A. 1999. A dithered K-S statistic. Commun. Stat. Theory Methods 28:1535-1546.

30. Wilks, D. S. 1995. Statistical Methods in the Atmospheric Sciences. Academic Press, San Diego.

31. Zarnoch, S. J., Anderson, R. L., and Sheffield, R. M. 1995. Using the beta-binomial distribution to characterize forest health. Can. J. For. Res. 25:462-469. 